Aerodynamik des Flugzeuges

Erster Band 


\section{Aerodynamik \\ des Flugzeuges}

Von

\section{Dr. phil. H. Schlichting}

o. Professor an der Technischen Hochschule Braunschweig Direktor der Aerodynamischen Versuchsanstalt Göttingen und Leiter des Instituts für Aerodynamik der Deutschen Forschungsanstalt für Luftfahrt Braunschweig

\section{Dr.-Ing. E.Truckenbrodt}

o. Professor für Technische Mechanik und Direktor des Instituts für Strömungsmechanik an der Technischen Hochschule München

\section{Erster Band}

Grundlagen aus der Strömungsmechanik Aerodynamik des Tragflügels (Teil I)

Mit 260 Abbildungen

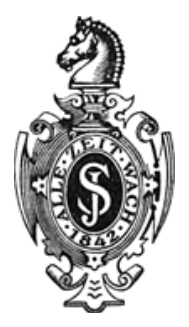

Springer-Verlag

Berlin / Göttingen / Heidelberg 
Alle Rechte, insbesondere das der Übersetzung in fremde Sprachen, vorbehalten Ohne ausdrückliche Genehmigung des Verlages ist es auch nicht gestattet, dieses Buch oder Teile daraus auf photomechanischem Wege

(Photokopie, Mikrokopie) zu vervielfältigen

$$
\begin{gathered}
\text { ISBN 978-3-642-53045-6 ISBN 978-3-642-53044-9 (eBook) } \\
\text { DOI 10.1007/978-3-642-53044-9 }
\end{gathered}
$$

(C) by Springer-Verlag OHG., Berlin/Göttingen/Heidelberg 1959

Softcover reprint of the hardcover 1st edition 1959

Die Wiedergabe von Gebrauchsnamen, Handelsnamen, Warenbezeichnungen usw. in diesem Buche berechtigt auch ohne besondere Kennzeichnung nicht zu der Annahme, daß solche Namen im Sinne der Warenzeichen- und Markenschutz-Gesetzgebung als frei zu betrachten wären und daher von jedermann benutzt werden dürften 


\section{Vorwort}

Die Grundlagen der Aerodynamik des Flugzeuges sind in einer ausführlichen Darstellung in deutscher Sprache zuletzt vor mehr als zwanzig Jahren in den bekannten Büchern von R. Fuchs und L. HopF des Springer-Verlages behandelt worden. Bei der außerordentlich raschen Entwicklung und der starken Ausweitung, welche dieses Gebiet in den letzten beiden Jahrzehnten erfahren hat, ist es verständlich, daß eine einfache Neubearbeitung der beiden Bände von Fuchs und Hopf unmöglich ist. Als vor nunmehr etwa fünf Jahren Herr Dr. Julius SPRINGER uns deshalb den Vorschlag machte, als Ersatz für den „FuchsHopf" ein völlig neues Lehrbuch über die Aerodynamik des Flugzeuges zu verfassen, haben wir diesen Plan nur sehr zögernd aufgegriffen. Denn damals war noch nicht abzusehen, ob die nach dem Ausgang des zweiten Weltkrieges zum Erliegen gekommene deutsche Flugzeugindustrie wieder aufleben würde, und ob auch eine deutsche Luftfahrtforschung wieder erstehen würde. Wenn wir uns schließlich doch dazu entschlossen, die sehr umfangreiche Arbeit der völligen Neufassung eines Werkes über die Aerodynamik des Flugzeuges zu übernehmen, so taten wir es deshalb, weil wir letztlich die Entwicklung der deutschen Flugzeugindustrie und der deutschen Luftfahrtforschung optimistisch beurteilten, und weil wir glaubten, daß für die Ausbildung des jungen Ingenieurnachwuchses ein umfassendes Lehrbuch auf diesem Gebiet unentbehrlich sein würde.

Wir waren uns von vornherein darüber klar, daß wegen des außerordentlich starken Anwachsens des Stoffes in den letzten beiden Jahrzehnten es uns nicht möglich sein würde, das gesamte Gebiet darzustellen, welches das zweibändige Werk von FucHs-HopF behandelte, nämlich die ,Theorie der Luftkräfte“ (R. Fuchs) und die „Mechanik des Flugzeuges" (L. Hopf). Wir entschlossen uns, nur die ,Theorie der Luftkräfte“" zu behandeln, und die Neuabfassung der ,Flugmechanik", d. h. die Bewegung des Flugzeuges bei gegebenen Luftkräften, einem anderen Autor zu überlassen. Auch die mit dem Antrieb des Flugzeuges im Zusammenhang stehenden aerodynamischen Fragen (Triebwerksaerodynamik) bleiben im vorliegenden Werk unberücksichtigt. Nachdem für den Antrieb des Flugzeuges das Strahltriebwerk und die Rakete neben der Luftschraube eine überragende Bedeutung erlangt haben, ist das Gebiet der Triebwerks-Aerodynamik so umfangreich, daß es gesondert behandelt werden muß. 
In dem vorliegenden Werk „Aerodynamik des Flugzeuges" beschäftigen wir uns ausschließlich mit den Luftkräften, welche bei der Bewegung des Flugzeuges durch die irdische Atmosphäre an seinen Teilen und damit am ganzen Flugzeug wirksam sind (Aerodynamik der Flugzeugzelle). Diese Luftkräfte hängen in recht verwickelter Weise von der geometrischen Gestalt des Flugzeuges, von der Fluggeschwindigkeit, von den Bewegungsformen des Flugzeuges und von einigen physikalischen Eigenschaften der Luft ab. Aufgabe der Aerodynamik des Flugzeuges ist es, über diese Zusammenhänge Auskunft zu geben. Die Aerodynamik des Flugzeuges bildet die unerläßliche Grundlage für die Flugmechanik und auch für viele Fragen der Festigkeit des Flugzeuges. Jedoch haben wir auf die Behandlung des umfangreichen Sondergebietes der instationären Luftkräfte (Aeroelastizität) verzichten müssen.

Das Studium der aerodynamischen Fragen des Flugzeuges erfordert sehr gründliche Kenntnisse in der Strömungslehre. Aus diesem Grunde haben wir es für angebracht gehalten, einen ziemlich ausführlichen $\mathrm{Ab}$ riß der allgemeinen Strömungslehre vorauszuschicken. Neben der klassischen Hydrodynamik des vorigen Jahrhunderts kommt dabei der modernen Strömungslehre dieses Jahrhunderts mit den drei Teilgebieten „Gasdynamik“, „Grenzschicht-Theorie“ und „Tragflügel-Theorie“ eine große Bedeutung zu. Diese Grundlagen der Strömungslehre sind nicht nur für die Luftfahrt, sondern auch für viele andere Gebiete der Physik und Technik von erheblichem Interesse.

Das vorliegende Werk gliedert sich in drei Hauptabschnitte mit insgesamt zwölf Kapiteln, die auf zwei Bände verteilt sind. Im ersten Abschnitt werden nach einem einleitenden Kapitel über die Atmosphäre die Grundlagen aus der Strömungsmechanik in einem solchen Umfang dargestellt, wie sie in den folgenden Abschnitten benötigt werden. Der zweite Abschnitt befaßt sich mit der Aerodynamik des Tragflügels und der dritte Abschnitt mit der Aerodynamik der übrigen Teile des Flugzeuges (Rumpf, Leitwerke), wobei auch die Probleme der gegenseitigen Beeinflussung der Flugzeugteile eine wichtige Rolle spielen. Der modernen Entwicklung im Flugzeugbau wird dadurch Rechnung getragen, daß die Aerodynamik bei hohen Geschwindigkeiten (Unter- und Überschallgeschwindigkeit) ziemlich eingehend behandelt wird.

Dieses Buch wendet sich in erster Linie an Ingenieure. Wir haben uns bemüht, soweit wie möglich die theoretische Behandlung der Probleme in den Vordergrund zu stellen. Dabei haben wir versucht, diese in eine anschauliche, für den Ingenieur leicht faßliche Form zu bringen. In der Tat ist es heute möglich, unter Verwendung der modernen Strömungslehre den überwiegenden Teil der Aerodynamik des Flugzeuges aus rein theoretischen Überlegungen zu gewinnen. Das in der 
Literatur vorhandene, sehr umfangreiche experimentelle Material wurde nur insoweit herangezogen, als es zur Belebung der physikalischen Anschauung und zur Nachprüfung der Theorie erforderlich ist. Es kam uns darauf an, zum Ausdruck zu bringen, daß die entscheidenden Fortschritte nicht durch eine Anhäufung von umfangreichen Versuchsergebnissen erreicht worden sind, sondern vielmehr durch die Synthese von theoretischen Überlegungen mit wenigen grundlegenden Experimenten. Darüber hinaus haben wir uns bemüht, die Ergebnisse der Theorie dem Leser durch zahlreiche durchgerechnete Beispiele näherzubringen.

Für den ersten Abschnitt des Buches (Grundlagen aus der Strömungsmechanik) konnten wir weitgehend auf Vorlesungsmanuskripte des ersten Verfassers zurückgreifen, u. a. auch auf das Buch „GrenzschichtTheorie", welches vor kurzem beim Verlag G. Braun, Karlsruhe, in dritter Auflage erschienen ist. Weiterhin kam es uns sehr zustatten, daß der zweite Verfasser durch seine langjährige Tätigkeit in der Luftfahrt, zuletzt als Leiter der aerodynamischen Abteilung der Heinkel-FlugzeugWerke, mit den Problemen des Flugzeugbaues in enger Berührung war.

Der hiermit vorgelegte erste Band umfaßt sechs Kapitel, nämlich den ersten Abschnitt „Grundlagen aus der Strömungsmechanik“ mit vier Kapiteln und zwei Kapitel aus dem zweiten Abschnitt „Aerodynamik des Tragflügels". Wir hoffen, daß es uns möglich sein wird, den zweiten Band bald folgen zu lassen.

Bei der Herstellung der Abbildungen und beim Lesen der Korrekturen wurden wir von unseren Mitarbeitern V. Denk, K. Gersten, R. Karwinski und W. PeChau wesentlich unterstützt. Die sorgfältige Herstellung der Reinschrift des Manuskriptes verdanken wir Fräulein M. Frobenius. Dem Springer-Verlag danken wir für sein bereitwilliges Eingehen auf unsere Wünsche sowie für die sorgfältige Ausstattung des Buches.

Göttingen, im August 1958

H. Schlichting E. Truckenbrodt 


\title{
Inhaltsverzeichnis
}

\author{
Teil A \\ Grundlagen aus der Strömungsmechanik
}

I. Einführung und physikalische Eigenschaften der Atmosphäre

1.1 Aufgaben der Flugzeug-Aerodynamik . . . . . . . . 1

1.2 Physikalische Eigenschaften der Luft. . . . . . . . . . 2

1.21 Strömungsmechanik und physikalische Eigenschaften des strömenden Mediums. . . . . . . . . . . . . . . . 2

1.22 Druck, Dichte und Temperatur in der Atmosphäre . . . . . . . 4

1.23 Kompressibilität . . . . . . . . . . . . . . . . . . . 8

1.24 Zähigkeit . . . . . . . . . . . . . . . . . 11

1.3 Ähnlichkeitsgesetze der Strömungen . . . . . . . . 13

Literatur. . . . . . . . . . . . . . . . 18

\section{Inkompressible reibungslose Strömungen (Hydrodynamik)}

2.1 Kinematik der Flüssigkeitsbewegungen . . . . . . . . . . 18

2.11 Darstellungsmethoden . . . . . . . . . . . . . . . . . . . . 19

2.12 Dreidimensionale, zweidimensionale und eindimensionale Bewegungen . . . . . . . . . . . . . . . . . . 21

2.13 Stromlinien und Stromröhren . . . . . . . . . . . . . 22

2.14 Kontinuitätsgleichung . . . . . . . . . . . . . . . . . 23

2.15 Drehung einer Flüssigkeitsbewegung . . . . . . . . . . . . 25

2.2 Eindimensionale Strömungen (Stromfadentheorie) . . . . . 29

2.21 Eindimensionale Eulersche Bewegungsgleichung. . . . . . . . 29

2.22 Bernodllische Gleichung . . . . . . . . . . . . . . . . . 34

2.23 Einige Anwendungen der Bernoullischen Gleichung. . . . . . 36

2.231 Ausfluß aus einem Gefäß . . . . . . . . . . . . . . . 36

2.232 Messung von Druck und Geschwindigkeit in einer Strömung 37

2.233 Trennungsflächen . . . . . . . . . . . . . . . . . . . 39

$2.3 \mathrm{Zwei-und} \mathrm{dreidimensionale} \mathrm{Strömungen} \mathrm{.} \mathrm{.} \mathrm{.} \mathrm{.} \mathrm{.} \mathrm{.} \mathrm{.} \mathrm{.} \mathrm{.} \mathrm{.} 40$

2.31 Die allgemeinen Eulerschen Bewegungsgleichungen . . . . . . 40

2.32 Bernoullische Gleichung . . . . . . . . . . . . . . . . . . 42

2.33 Drehungsfreie Strömungen als Lösungen der EvLERschen Bewegungsgleichungen . . . . . . . . . . . . . . . 44 44

2.34 Potential- und Stromfunktion . . . . . . . . . . . . . . . . 46

2.35 Beispiele einfacher Potentialströmungen . . . . . . . . . . 51

2.351 Translationsströmung . . . . . . . . . . . . . . 52

2.352 Ebene Staupunktströmung. . . . . . . . . . . . . 52

2.353 Rotationssymmetrische Staupunktströmung ... . . . . . 53 
Seite

2.354 Ebene Quell- und Senkenströmung . . . . . . . . . . 54

2.355 Ebener Potentialwirbel . . . . . . . . . . . . . 55

2.356 Räumliche Quell- und Senkenströmung . . . . . . . . 57

2.357 Strömung um einen ebenen Halbkörper. . . . . . . . . 57

2.358 Strömung um einen rotationssymmetrischen Halbkörper . 60

2.359 Dipol und Kreiszylinder . . . . . . . . . . . . . . 61

2.3510 Strömung um eine Kugel. . . . . . . . . . . . . . . 67

2.3511 Strömung um andere Körper . . . . . . . . . . . . . 68

2.4 Wirbelbewegung . . . . . . . . . . . . . . . . . . . 70

2.41 Begriff der Zirkulation . . . . . . . . . . . . . . . . 70

2.42 Beispiele für Strömungen mit Zirkulation. . . . . . . . . . . 73

2.421 Translationsströmung . . . . . . . . . . . . . . . . 73

2.422 Translationsströmung mit Trennungsfläche. . . . . . . . 73

2.423 Potentialwirbel . . . . . . . . . . . . . . . . . . 74

2.424 Tragflügel mit Auftrieb . . . . . . . . . . . . . . . . 75

2.43 Zusammenhang zwischen Zirkulation und Drehung. . . . . . . 75

2.44 Dynamik der Wirbelbewegung . . . . . . . . . . . . . . . 77

2.45 Satz von W. THomson . . . . . . . . . . . . . . . . . . . 79

2.46 Entstehung der Zirkulation bei der Anfahrt eines Tragflügels . . 81

2.47 Die Helmholtzschen Wirbelsätze . . . . . . . . . . . . . . 83

2.48 Das Biot-Savartsche Gesetz . . . . . . . . . . . . . . . . 85

2.5 Berechnung ebener Potentialströmungen mit Hilfe komplexer Funktionen. . . . . . . . . . . . . . . 91

2.51 Die Grundgleichungen. . . . . . . . . . . . . . . . . 91

2.52 Die CadchY-Riemannschen Differentialgleichungen. . . . . . . 92

2.53 Die komplexe Strömungsfunktion . . . . . . . . . . . . . . 93

2.54 Beispiele zur komplexen Strömungsfunktion. . . . . . . . . . 94

2.541 Translationsströmung . . . . . . . . . . . . . . 95

2.542 Strömung in einem Winkelraum . . . . . . . . . . . . 95

2.543 Quelle, Senke und Potentialwirbel . . . . . . . . . . 97

2.544 Dipol . . . . . . . . . . . . . . . . . . . . . . . . 98

2.545 Translationsströmung um den Kreiszylinder . . . . . . . 99

2.546 Strömung um den Kreiszylinder mit Zirkulation . . . . . 100

2.55 Der Begriff der konformen Abbildung . . . . . . . . . . . . 103

2.56 Beispiele zur konformen Abbildung. . . . . . . . . . . . . . 107

2.561 Die parallel angeströmte Platte. . . . . . . . . . . . . 107

2.562 Die senkrecht angeströmte Platte. . . . . . . . . . . . 108

2.563 Die ebene angestellte Platte . . . . . . . . . . . . . 110

2.564 Elliptische Zylinder . . . . . . . . . . . . . . . . . . 119

2.565 Joukowsky-Profile . . . . . . . . . . . . . . . . 122

2.6 Der Impulssatz. . . . . . . . . . . . . . . . . . . . 124

2.61 Das allgemeine Theorem des Impulssatzes . . . . . . . . . . 124

2.62 Beispiele zum Impulssatz . . . . . . . . . . . . . . . . . . 128

2.621 Strömung in einer Rohrumlenkung . . . . . . . . . . . . 128

2.622 Der Strahl senkrecht auf eine Wand . . . . . . . . . . 129

2.623 Der Strahl schräg auf eine Wand . . . . . . . . . . . 130

2.624 Widerstand eines Halbkörpers . . . . . . . . . . . . . . 131

2.625 Ermittlung des Widerstandes aus dem Impulsverlust. . . 132

Literatur . . . . . . . . . . . . . . . . 136 


\section{Kompressible reibungslose Strömungen (Gasdynamik)}

3.1 Grundbegriffe . . . . . . . . . . . . . . 136

3.11 Kompressibilität, MacHsche Zahl . . . . . . . . . . . . 136

3.12 Zustandsgleichung . . . . . . . . . . . . . . . . . . . . 138

3.13 Schallgeschwindigkeit . . . . . . . . . . . . . . . . 139

3.14 MACHscher Winkel . . . . . . . . . . . . . . . . . . . 141

3.15 Temperaturerhöhung in einer kompressiblen Strömung . . . . . 144

3.2 Eindimensionale Strömungen (Stromfadentheorie) . . . . . 146

3.21 Eulersche Bewegungsgleichung und Bernoullische Gleichung . 146

3.22 Kritische MaCH-Zahl . . . . . . . . . . . . . . . . . . . . 149

3.23 Staupunkt. . . . . . . . . . . . . . . . . . . 151

3.24 Ausfluß aus einem Kessel . . . . . . . . . . . . . . . . . . 153

3.25 Lavaldüse . . . . . . . . . . . . . . . . . . . . . . . . 158

3.3 Zweidimensionale Strömungen. . . . . . . . . . . . 159

3.31 Grundgleichungen. . . . . . . . . . . . . . . . . . . . 159

3.32 Drehungsfreiheit . . . . . . . . . . . . . . . . 160

3.33 Geschwindigkeitspotential . . . . . . . . . . . . . . 161

3.34 Lösungstypus für Überschallströmungen . . . . . . . . . . . 163

3.35 Strömung längs einer schwach welligen Wand . . . . . . . 164

3.4 Ebene Unterschallströmungen . . . . . . . . . . . . . . 167

3.41 Verfahren von JANZEN und RAYLEIGH . . . . . . . . . . . . 167

3.42 Prandtl-Gladertsche Regel . . . . . . . . . . . . . . . . . 170

3.43 Vergleich mit Versuchsergebnissen . . . . . . . . . . . . . 175

3.5 Ebene Ưberschallströmungen . . . . . . . . . . . . . 176

3.51 Prandtl-Meyersche Eckenströmung . . . . . . . . . . . . . 176

3.52 Strömung um eine flache Ecke . . . . . . . . . . . . . . . 182

3.53 Auftrieb und Widerstand der ebenen Platte. . . . . . . . . . 185

3.54 Auftrieb und Widerstand schlanker Profile . . . . . . . . . . 188

3.55 Charakteristiken-Verfahren für Überschallströmungen um Profile . 195

3.6 Ebene transsonische Strömungen . . . . . . . . . . . . 196

3.61 Allgemeines und experimentelle Ergebnisse . . . . . . . . . 196

3.62 Der senkrechte Verdichtungsstoß . . . . . . . . . . . . . . . 204

3.63 Der schiefe Verdichtungsstoß. . . . . . . . . . . . . . . . . 207

3.64 Das Stoßpolaren-Diagramm . . . . . . . . . . . . . . . 211

3.65 Von KáRMánsches Ähnlichkeitsgesetz. . . . . . . . . . . . . 213

Literatur. . . . . . . . . . . . . . . . . . . 217

\section{Strömungen mit Reibung (Grenzschicht-Theorie)}

4.1 Grundzüge der Strömung mit Reibung . . . . . . . . . . . 219

4.11 Allgemeines . . . . . . . . . . . . . . . . . . . . . 219

4.12 NewTonsches Reibungsgesetz . . . . . . . . . . . . . . 220

4.13 REYNoLDssches Ähnlichkeitsgesetz . . . . . . . . . . . . . . 220

4.14 Laminare Rohrströmung . . . . . . . . . . . . . . . . . . 222

4.15 Turbulente Rohrströmung . . . . . . . . . . . . . . . . . . 226

4.16 Das Widerstandsproblem umströmter Körper . . . . . . . . . 231

4.2 Grundzüge der Grenzschicht-Theorie . . . . . . . . . . . 235

4.21 Begriff der Grenzschicht . . . . . . . . . . . . . . 235

4.22 Ablösung der Grenzschicht . . . . . . . . . . . . . . 236 
4.23 Abschätzung der Grenzschichtdicke und des Reibungswiderstandes bei laminarer Strömung . . . . . . . . . . . . . . . . 240

4.24 Turbulente Strömung in der Grenzschicht . . . . . . . . . . 241

4.3 Die Bewegungsgleichungen der zähen Flüssigkeit (NavierStokessche Gleichungen). . . . . . . . . . . . . . 243

4.4 Die Frandtlschen Grenzschichtgleichungen . . . . . . . . 247

4.41 Aufstellung der Grenzschichtgleichungen . . . . . . . . . . . 247

4.42 Einige physikalische Eigenschaften der Grenzschicht. . . . . . 249

4.43 Die Plattengrenzschicht bei laminarer Strömung. . . . . . . . 251

4.44 Impulssatz der Grenzschicht. . . . . . . . . . . . . . . . . 255

4.45 Näherungsverfahren zur Berechnung der laminaren Grenzschicht 257

4.5 Grenzschichtbeeinflussung. . . . . . . . . . . 260

4.51 Allgemeines . . . . . . . . . . . . . . . . . . . . . 260

4.52 Mitbewegen der Wand . . . . . . . . . . . . . . . 261

4.53 Beschleunigung der Grenzschicht. . . . . . . . . . . . . . 261

4.54 Absaugung der Grenzschicht. . . . . . . . . . . . . . 264

4.55 Laminarhaltung durch Formgebung (Laminarprofile) . . . . 271

4.6 Einiges über turbulente Strömungen . . . . . . . . . . . . 272

4.61 Mittlere Bewegung, Schwankungsbewegung und turbulente Schein-

reibung . . . . . . . . . . . . . . . . . . 272

4.62 Windkanalturbulenz. . . . . . . . . . . . . . . . . . . 275

4.63 Der Prandtlsche Mischungsweg . . . . . . . . . . . . . . . 276

4.64 Geschwindigkeitsverteilung in der turbulenten Grenzschicht. . . 278

4.7 Der turbulente Reibungswiderstand der längsangeströmten

ebenen Platte . . . . . . . . . . . . . . . 280

4.71 Die glatte Platte bei inkompressibler Strömung . . . . . . . 280

4.72 Einfluß der Kompressibilität. . . . . . . . . . . . . . . . . 284

4.73 Einfluß der Rauhigkeit . . . . . . . . . . . . . . . . . . 286

4.8 Berechnung der Grenzschicht mit Druckabfall und Druckanstieg . . . . . . . . . . . . . . . 291

4.81 Allgemeines . . . . . . . . . . . . . . . . . . . . . . . . 291

4.82 Kenngrößen der Grenzschicht . . . . . . . . . . . . . . . . 292

4.83 Berechnung der Impulsverlustdicke. . . . . . . . . . . . . . 295

4.84 Beispiel . . . . . . . . . . . . . . . . . . . . . . . . . . 296

4.85 Rechnerische Ermittlung des Profilwiderstandes . . . . . . . 298

4.9 Grenzschichten bei kompressibler Strömung . . . . . . . 301

4.91 Allgemeines . . . . . . . . . . . . . . . . . . 301

4.92 Stoffbeiwerte. . . . . . . . . . . . . . . . . . . . . . 302

4.93 Grundgleichungen . . . . . . . . . . . . . . . . . . . . . . 303

4.94 Temperaturerhöhung durch adiabatische Kompression und Rei-

bung ................ . . 304

4.95 Laminare Plattengrenzschicht . . . . . . . . . . . . . . . . 308

4.96 Zusammenwirken von Grenzschicht und Verdichtungsstoß . . . . 309

4.10 Der Umschlag laminar-turbulent . . . . . . . . . . . . . . 312

4.101 Experimentelle Ergebnisse . . . . . . . . . . . . . . . . 312

4.102 Grundzüge der Stabilitätstheorie der Laminarströmung . . . . 314

4.103 Ermittlung des Umschlagpunktes für ein Tragflügelprofil . . 319

Literatur . . . . . . . . . . . . . . . . . . . . . 322 


\section{Teil B}

\section{Aerodynamik des Tragflügels}

\section{Einführung in die Aerodynamik des Tragflügels}

5.1 Geometrie des Tragflügels . . . . . . . . . . . . . 327

5.11 Allgemeine Angaben . . . . . . . . . . . . . . . . . . . . 327

5.12 Flügelgrundriß . . . . . . . . . . . . . . . . . . 328

5.13 Flügelprofil . . . . . . . . . . . . . . . . . 332

5.14 Verwindung und V-Stellung . . . . . . . . . . . . 340

5.15 Ausgeführte Flügelformen . . . . . . . . . . . . . . . . . . 341

5.2 Kräfte und Momente am Tragflügel . . . . . . . . . . . . 349

5.21 Auftrieb, Widerstand und Gleitzahl. . . . . . . . . . . . . 349

5.22 Sonstige Kräfte und Momente, Achsensysteme. . . . . . . . . 350

5.23 Dimensionslose Beiwerte der Kräfte und Momente. . . . . . . 352

5.24 Druckverteilungen und Auftriebsverteilungen . . . . . . . . . 354

5.3 Zusammenhang zwischen den Luftkräften und den Bewegungs-

formen des Tragflügels. . . . . . . . . . . . . 357

5.31 Bewegungsformen des Flugzeuges . . . . . . . . . . . . . 357

5.32 Kräfte und Momente beim Geradeausflug . . . . . . . . . . . 3599

5.33 Kräfte und Momente beim Schiebeflug . . . . . . . . . . . . 361

5.34 Kräfte und Momente bei Drehbewegungen des Flugzeuges . . . 362

Literatur . . . . . . . . . . . . . . . . . . . . . 363

VI. Der Tragflügel unendlicher Spannweite bei inkompressibler Strömung (Profiltheorie)

6.1 Grundlagen der Theorie des Auftriebes . . . . . . . . . 364

6.11 Allgemeines . . . . . . . . . . . . . . . . . . . . . . . 364

6.12 Der Satz von Kutta-Joukowsky . . . . . . . . . . . . . 366

6.121 Berechnung des Auftriebes aus dem Druckintegral . . . 366

6.122 Berechnung des Auftriebes für ein Flügelgitter . . . . . . . . 367

6.13 Entstehung und Größe der Zirkulation . . . . . . . . . . . . 371

6.2 Profiltheorie nach der konformen Abbildung . . . . . . . 374

6.21 Allgemeines ......... . . . . . . . . . . . . 374

6.22 Berechnung von Auftrieb und Moment für ein beliebiges Tragflügelprofil . . . . . . . . . . . . . . . . . 375

6.221 Die Blasiusschen Formeln . . . . . . . . . . . . 375

6.222 Anderer Beweis der Kutta-Joukowskyschen Formel. . . 378

6.223 Nullauftriebsrichtung eines Profils (erste Achse) . . . . . 379

6.23 Kreisbogenprofil sehr geringer Dicke . . . . . . . . . . . . . 382

6.24 Symmetrisches Joukowsky-Profil . . . . . . . . . . . . . . 386

6.25 Schlußbemerkung. . . . . . . . . . . . . . . . . 389

6.3 Profiltheorie nach der Singularitätenmethode. . . . . . 390

6.31 Allgemeines . . . . . . . . . . . . . . . . . . . . . . . 390

6.32 Sehr dünne Profile (Skelett-Theorie) . . . . . . . . . . . . . 391

6.321 Grundlagen der Skelett-Theorie. . . . . . . . . . . . . 391

6.322 Berechnung der Skelettlinie aus der Zirkulationsverteilung

(I. Hauptaufgabe) . . . . . . . . . . . . . . . . . . . 394 
6.323 Berechnung der aerodynamischen Beiwerte . . . . . . . 399

6.324 Berechnung der Geschwindigkeitsverteilung auf der Profilkontur (II. Hauptaufgabe) . . . . . . . . . . . . . . . 407

6.33 Symmetrische Profile endlicher Dicke bei symmetrischer Anströmung (Tropfentheorie) . . . . . . . . . . . . . . 411

6.331 Grundlagen der Tropfentheorie . . . . . . . . . . . . 411

6.332 Berechnung der Geschwindigkeitsverteilung auf der Profilkontur . . . . . . . . . . . . . . . . . . . 413

6.333 Berechnung des Profiltropfens aus der vorgegebenen Geschwindigkeitsverteilung ..... . . . . . . . 417

6.34 Profile endlicher Dicke mit Anstellwinkel . . . . . . . . . . . 420

6.341 Berechnung der Geschwindigkeitsverteilung auf der Profilkontur . . . . . . . . . . . . . . . . . . . . . . 420

6.342 Berechnung der aerodynamischen Beiwerte . . . . . . 422

6.343 Berechnung der Profilform aus der vorgegebenen Geschwindigkeitsverteilung . . . . . . . . . . . . . . . . . . . 429

6.35 Das Geschwindigkeitsfeld in der Umgebung eines Tragflügels . . . 431

6.36 Der Tragflügel in gekrümmter Strömung . . . . . . . . . . . 434

6.4 Einfluß der Reibung auf die Profileigenschaften . . . . . 437

6.41 Auftrieb ... . . . . . . . . . . . . . . 437

6.42 Widerstand . . . . . . . . . . . . . . . . . . . 441

Literatur . . . . . . . . . . . . . . . . . 445

Namenverzeichnis . . . . . . . . . . . . . . . . . . 448

Sachverzeichnis ................... . . 451 


\section{Zusammenstellung der wichtigsten Formelgrößen}

Wegen der Vielzahl der auftretenden Größen war es nicht vermeidbar, daß einige Buchstaben in mehrfacher Bedeutung verwendet werden. So bedeutet z. B. $\lambda$ in Kap. 4.1 die Rohrwiderstandszahl, in Kap. 4.9 die Wärmeleitzahl und in Kap. 5 die Zuspitzung des Flügels.

Im folgenden sind die wichtigsten Bezeichnungen zusammengestellt:

\section{Stoffbeiwerte}

$\gamma$

$\varrho=\gamma / g$ Dichte (Masse der Volumeneinheit)

$g \quad$ Erdbeschleunigung

$c_{p}, c_{v}$ spezifische Wärme bei konstantem Druck bzw. konstantem Volumen

$x=c_{p} / c_{v}$ Adiabaten-Exponent

$a=\sqrt{\varkappa p / \varrho}$ Schallgeschwindigkeit

$R \quad$ Gaskonstante

$\mu \quad$ Zähigkeitsbeiwert

$\nu=\mu / \varrho$ kinematische Zähigkeit

$\lambda$ Wärmeleitzahl

$a_{1} \quad$ Temperaturleitfähigkeit

$\operatorname{Pr}=v / a_{1}=\mu g c_{p} / \lambda$ Prandtl-Zahl

\section{Strömungsgrößen}

$p$ Druck (Normalkraft pro Flächeneinheit)

$\tau \quad$ Schubspannung (Tangentialkraft pro Flächeneinheit)

$u, v, w$ Geschwindigkeitskomponenten in rechtwinkligen Koordinaten

$w_{r}, w_{\varphi}$ Geschwindigkeitskomponenten in Polarkoordinaten

$V, U_{\infty}, w_{\infty}, u_{\infty}$ Anströmungsgeschwindigkeit

$u_{K}, W_{K}$ Geschwindigkeit auf der Profilkontur

$q=\frac{\varrho}{2} V^{2}$ Staudruck

$T$ absolute Temperatur
$\mathrm{Re}=V l / \nu$ REYNOLDSsche Zahl

$\mathrm{Ma}=V / a$ Machsche Zahl

$\mu \quad$ Machscher Winkel

$\delta \quad$ Grenzschichtdicke

$\delta^{*} \quad$ Verdrängungsdicke der Grenzschicht

$\vartheta \quad$ Impulsverlustdicke der Grenzschicht

$\Phi$ Geschwindigkeitspotential

$\Psi \quad$ Stromfunktion

$\Gamma \quad$ Zirkulation

$k(x)$ Wirbeldichte

$q(x)$ Quelldichte

$\lambda$ Rohrwiderstandszahl

\section{Geometrische Größen}

$x, y, z$ rechtwinklige Koordinaten

$z=x+i y$ komplexe $\cdot$ Koordinate

$r, \varphi$ Polarkoordinaten

$D=2 R$ Durchmesser

$l \quad$ Körperlänge, Flügeltiefe

$b=2 s$ Flügelspannweite

$F \quad$ Flügelfläche

$\Lambda=b^{2} / F$ Seitenverhältnis des Flügels

$l_{\boldsymbol{i}}, l_{\boldsymbol{a}}$ Flügeltiefe innen bzw. außen

$\lambda=l_{a} / l_{i}$ Flügelzuspitzung

$\varphi \quad$ Pfeilwinkel

$\varepsilon \quad$ Verwindungswinkel

$v \quad$ Winkel der $V$-Stellung

$N_{25}$ geometrischer Neutralpunkt

$d \quad$ Profildicke

$\delta=d / l$ relatives Dickenverhältnis 
$f \quad$ Profilwölbung

$M \quad$ Kippmoment

$x_{d} \quad$ Dickenrücklage

$N \quad$ Giermoment

$x_{f} \quad$ Wölbungsrücklage

$c_{A} \quad$ Auftriebsbeiwert, Gl. (5.39)

$z^{(s)}, z^{(t)}$ Skelettlinie bzw. Profiltropfen

$c_{W} \quad$ Widerstandsbeiwert

4. Aerodynamische Größen

$c_{Y} \quad$ Seitenkraftbeiwert

(Vgl. Abb. 5.14)

$\alpha \quad$ Anstellwinkel

$c_{L} \quad$ Rollmomentenbeiwert

$c_{M}$ Kippmomentenbeiwert

$\beta \quad$ Schiebewinkel

$c_{N} \quad$ Giermomentenbeiwert

$A$ Auftrieb

$W \quad$ Widerstand

$Y \quad$ Seitenkraft

$L \quad$ Rollmoment

$c_{p}=\left(p-p_{\infty}\right) / q$ Druckbeiwert

$\Delta c_{p} \quad$ Beiwert der Lastverteilung

$\varepsilon \quad$ Gleitwinkel

c) Beiwert des Reibungswiderstandes 